\section{Cómo encontrar un lugar en el mundo: explorando experiencias de recuperación de personas con trastornos mentales graves}

\section{How to find a place in the world: exploring people's experiences recovering from serious mental disorders}

\author{
Francisco Javier Saavedra Macías \\ Professor do Departamento de Psicología Experimental/ \\ Universidad de Sevilla. \\ Universidad de Sevilla \\ Dept. Psicología Experimental \\ $\mathrm{C} /$ Camilo José Cela s/n \\ 41018 - Sevilla - España \\ fjsaavedra@us.es
}

Recebido para publicação em setembro de 2009. Aprovado para publicação em outubro de 2010.
SAAVEDRA MACÍAS, Francisco Javier.

Cómo encontrar un lugar en el mundo: explorando experiencias de recuperación de personas con trastornos mentales graves. História Ciências, Saúde - Manguinhos, Rio de Janeiro, v.18, n.1, jan.-mar. 2011, p.121-139

\section{Resumen}

Se realiza una revisión histórica, conceptual y cultural del concepto de recuperación en el contexto de la salud mental. Se revisan y discuten los resultados de los trabajos realizados en la última década sobre experiencias subjetivas de personas con trastornos mentales graves en proceso de recuperación y se analizan las diferencias entre los conceptos de rehabilitación, integración y recuperación. Tras una introducción histórica, la descripción de las experiencias de recuperación se ordenan en tres apartados: identidad y control de la propia vida; relaciones personales y comunidad; y recursos materiales, trabajo y otras actividades sociales. El concepto de recuperación implica nuevos retos y tensiones que los procesos de reforma de los sistemas de salud mental en Europa y América deben asumir.

Palabras claves: recuperación; trastornos mentales graves; narrativas.

Abstract

This historical, conceptual, and cultural review of the concept of recovery within the realm of mental health discusses the last decade's research into the subjective experiences of people in the process of recovering from serious mental disorders and analyzes the differences between the notions of rehabilitation, integration, and recovery. After presenting a historical introduction, the article describes recovery experiences from three angles: identity and control over one's own life; personal and community relationships; and material resources, work, and other social activities. The concept of recovery entails new challenges and tensions that must be taken into account in the reform of mental health systems in Europe and the Americas.

Keywords: recovery; serious mental disorders; narratives. 


\section{Rehabilitación, integración y recuperación: una introducción histórica}

Rehabilitación e integración son dos conceptos que han guiado la organización de los servicios comunitarios de salud mental en los diversos recorridos, algunos más exitosos que otros, que incluyen las reformas psiquiátricas en Europa y América. El concepto de rehabilitación psicosocial en salud mental surge a partir de importantes cambios políticos, sociales y científicos en los planteamientos de la gestión de la enfermedad mental (López, Laviana, 2007). Gracias a algunos modelos integradores de las décadas de los setenta y ochenta sobre la enfermedad mental, descubrimos que las necesidades de las personas que padecen esquizofrenia son múltiples y muy extensas y en ningún caso quedan satisfechas mediante un adecuado tratamiento farmacológico. Entre los distintos modelos clásicos, el modelo que probablemente más ha influenciado e impulsado la rehabilitación psicosocial del enfermo mental es el Modelo de la Vulnerabilidad de Zubin y Spring (1977); seguidamente vinieron otros que matizaban o profundizaban en algunos aspectos como el Modelo de Vulnerabilidad Estrés (Nuechterlein, Dawson, 1984) o el Modelo de Ciompi (1987). Liberman (1986) construyó un modelo integrado multifactorial de los trastornos mentales graves (TMG) y sus incapacidades. También la construcción de Emoción Expresada (EE; Brown, Rutter, 1966) ha sido esencial en el desarrollo de programas psicoeducativos y en la investigación sobre los factores psicosociales. Los programas de rehabilitación tienen como objetivo dotar a los pacientes de las habilidades y las competencias psicosociales para interactuar de forma autónoma en sociedad. Es evidente que el modelo manicomial aislaba a gran parte de las personas que padecían TMG concentrándolas en instituciones apartadas del resto del sistema sanitario, impidiendo que el modelo rehabilitador adquiriera su verdadera potencialidad (Vizeu, 2005). En Europa y América, la inclusión de los servicios de salud mental en el sistema sanitario normalizado mediante el modelo de atención comunitario ha logrado acercar a las personas que padecen TMG al resto de la población. Esta normalización del tratamiento, aunque tomada como un objetivo esencial en todas las declaraciones oficiales - en América, por ejemplo, mediante la famosa Declaración de Caracas de 1990 que estuvo auspiciada por la Conferencia Regional de la OMS sobre la Reestructuración de la Atención Psiquiátrica en América Latina - ha tenido éxito diverso. Mientras que el $80 \%$ de las camas psiquiátricas en América Latina y el Caribe se encuentran en hospitales psiquiátricos (Rodríguez, 2007), en la región Autónoma de Andalucía (España) no existe ningún hospital psiquiátrico y todos los servicios psiquiátricos están integrados en la atención médica normalizada. Es de destacar el esfuerzo de algunos países como Brasil que han disminuido sus camas en hospitales psiquiátricos en un 30\% creando, al mismo tiempo, nuevos servicios comunitarios como los Centros de Atención Psicosocial (Caps; Larrobla, 2007). En la actualidad existen más de mil Caps regulados por la disposición 366, de 19 de febrero de 2002 del Ministerio de Salud.

Integrar el tratamiento psiquiátrico en la atención sanitaria normalizada es la primera acepción del término integración. También podemos ser más ambiciosos y referirnos al objetivo final de todo proceso de rehabilitación: la integración social completa del paciente. Es decir, no basta con la normalización de la asistencia a personas que padecen TMG mediante su integración en los servicios sanitarios y sociales normalizados, sino que tenemos 
que lograr hacer participar de todos los intercambios sociales que se dan en la comunidad, como sujetos de derecho, a las personas que padecen un TMG. En este sentido, la Conferencia Regional sobre la Reforma de los Servicios de Salud Mental de Brasília, convocada 15 años después de la declaración de Caracas, enfatizó la necesidad de ir más allá de la simple normalización de los servicios de salud mental al integrarlos en la comunidad, al afirmar que era imprescindible "la promoción y la protección de los derechos humanos y civiles de las personas con problemas de salud mental" (Caldas de Almeida, 2005, p.23). Una prueba de este proceso de normalización es la inclusión de la cuestión de la reforma de los sistemas de salud mental y de la promoción y defensa de los derechos civiles de las personas con TMG en Latino-América en organizaciones como el Mercosur, que no están originariamente relacionadas con la salud mental. La promoción de los derechos civiles de las personas con trastornos mentales es una cuestión que incumbe no sólo a instituciones sanitarias, sino económicas y políticas. De hecho, el Foro de Autoridades de Salud Mental de los Países de América del Sur, celebrado del 3 al 5 de Septiembre de 2007 en Luján, Argentina, gestionó la inclusión del componente de salud mental en el Mercosur.

Más allá de estos procesos de normalización e integración, el objetivo del presente artículo es dar a conocer un nuevo concepto en el ámbito de la salud mental: recuperación. También se realizará una revisión de algunas investigaciones muy relevantes llevadas a cabo en la última década, desde esta perspectiva.

Mientras que el concepto de recuperación es central en el diseño de cualquier recurso socio-sanitario de salud mental en el mundo anglo-sajón (Mind, 2008; CSIP, RCPsych, SCIE, 2007; The Future Vision Coalition, 2009), en España y en América, aunque conocido y citado en algunos lugares (López, Laviana, 2007), no ha recibido en mi opinión la justa atención y desde luego no tiene un lugar privilegiado entre los gestores de los sistemas de salud mental. Una muestra de esto puede ser que en el pasado Plan Andaluz Integral de Salud Mental 2003-2008 (Junta de Andalucía. Consejería de Salud, 2003) no se encuentra el concepto recuperación ni una sola vez. En el reciente Plan Andaluz 2008-2012 (Junta de Andalucía, 2008) se aboga, sin embargo, por ir incorporando esta perspectiva en la gestión de la salud mental en Andalucía. En la amplia revisión de la reforma psiquiátrica en América en "La reforma de los servicios de salud mental: 15 años después de la Declaración de Caracas" se utiliza el término recuperación bajo un gran número de acepciones y en ningún momento se define el concepto contrastándolo con los más habituales de rehabilitación e integración. Por ello, considero esencial acercar de forma sintética y concisa este concepto, apuntar sus diferencias con otros de uso más común y describir algunas de las investigaciones desarrolladas desde esta perspectiva.

El Sainsbury Centre for Mental Health define la recuperación como

una serie de valores sobre los derechos de la persona a construir una vida significativa para sí misma, con o sin la presencia continua de síntomas de salud mental. La recuperación está basada en las ideas de la autodeterminación y el control de sí mismo. Hace énfasis en la importancia de la esperanza en el mantenimiento de la motivación y la ayuda con el objetivo de la consecución de una vida llena de sentido (The Future Vision Coalition, 2009, p.22). 
El concepto de recuperación presenta un origen muy especial. Para empezar, el foco de creación del concepto de recuperación como punto de partida del abordaje socio-sanitario de las personas que padecen TMG no corresponde a los profesionales de salud mental, ni tan siquiera a los políticos o a las asociaciones de familiares (Shepherd, Boardman, Slade, 2008). Aunque no podemos excluir a estas últimas de los movimientos que han hecho posible la consolidación del concepto de recuperación, el punto de partida para la aplicación del concepto de recuperación a los TMG es reciente (décadas de los 1990-2000) y se encuentra en las narrativas, autobiografías, informes, en definitiva en las experiencias subjetivas de personas con TMG, los cuales nos cuentan sus procesos de recuperación y nos demuestran que, a pesar de la imagen ominosa de los TMG, es posible disfrutar de una vida llena de sentido y conectada socialmente. Por lo tanto, son los movimientos de "clientes de los servicios de salud mental" y las "iniciativas reivindicadoras de los derechos sociales" las que han acercado el concepto de "recuperación" a nuestro sistema de salud mental (Jacobson, Curtis, 2000). ${ }^{1}$ La posición de estos grupos es muy crítica con el sistema de atención psiquiátrica, hasta el punto que se auto definen como supervivientes del sistema y se consideran traumatizados por el modelo biomédico. En la red se pueden encontrar páginas web de estos grupos. ${ }^{2}$ Por lo tanto, una característica básica de la inclusión del concepto de recuperación en los sistemas de salud mental es la reivindicación de la esperanza. Supone enfrentarse a lo que puede considerarse una segunda estigmatización desde el modelo biomédico, en la cual la idea de la recuperación en pacientes de TMG es casi herética (Ridgway, 2001).

Acercarse a la salud mental desde la perspectiva de la recuperación supone privilegiar el concepto de healing (sanar) frente al de cure (curar) (Jacobson, Greenley, 2001). La recuperación no requiere necesariamente la vuelta al estado de salud y funcionamiento anterior a la enfermedad. El proceso de recuperación implica la construcción de la identidad, integrando la enfermedad de tal forma que permita que otros componentes de aquélla no sólo se desarrollen, sino que, si es posible, la misma experiencia de la enfermedad adquiera algún sentido y pueda conectarse con otros componentes identitarios. Es decir, que los roles relacionados con la enfermedad no dominen la identidad de la persona. La percepción del propio control, como veremos posteriormente, es otro componente esencial de la recuperación. Y esto es así en dos sentidos: como estrategias y recursos para manejar los síntomas y el estrés y también como locus de control. Es decir, el grado en que los pacientes perciben que su situación depende de sí mismos. El control está conectado con el concepto de empowerment. Es decir, con la toma del poder, la adquisición de habilidad para actuar como un agente independiente y autónomo, la toma de responsabilidades y la capacidad para aceptar riesgos y hablar con una voz propia por parte de las personas que padecen TMG (Jacobson, Greenley, 2001). Es el concepto consumer, que se entiende como consumidor o usuario de recursos sociosanitarios, el elegido para identificar a las 'personas que padecen TMG' dentro del sistema de salud mental en la literatura anglosajona. En mi opinión el concepto de consumer está relacionado con la importancia del mundo del consumo y por la mejor aceptación social y estatus de la figura del consumidor o del cliente en nuestra sociedad. El modelo recuperador hace énfasis en la organización sociosanitaria y en, especialmente, en las prácticas profesionales, algo que las otras perspectivas no lo hacen 
con el mismo énfasis. En este sentido define una cultura positiva de healing como unos servicios orientados a la recuperación y la aceptación de los derechos humanos como guía de las decisiones políticas, las cuales deben promover la distribución del poder y los recursos de forma equitativa como factores externos a la recuperación. Al contrario de las investigaciones e intervenciones clásicas en rehabilitación psicosocial, las investigaciones relacionadas con el concepto de recuperación utilizan una metodología esencialmente cualitativa por medio de entrevistas narrativas abiertas o informes escritos como diarios, informes, cartas. El objetivo es privilegiar las experiencias subjetivas de éstos y permitir conocer los recursos que se utilizan para superar los problemas relativos a la enfermedad y el estigma. En Cuadro 1 se resumen las diferencias entre estos tres conceptos. Es necesario remarcar que los rasgos del concepto de recuperación que se indican en esta tabla no son incompatibles con los distintos rasgos de los conceptos de rehabilitación e integración. En el cuadro se señalan los rasgos más característicos de cada concepto y que conforman, en último término, modelos de gestión de la salud mental con distintos acentos.

El concepto de recuperación ha supuesto la reconsideración de los servicios de salud mental e incluso, en algunos países anglosajones, han conllevado su reforma. En un sentido distinto a los servicios orientados al tratamiento o a la cura, los servicios orientados a la recuperación apuestan por el empoderamiento y el control de los usuarios de sus interacciones con los servicios socio-sanitarios (Sainsbury Centre for Mental Health, 2009). Podemos encontrar guías para la implementación de servicios orientados a la recuperación en Irlanda, Inglaterra y EUA. Entre otras innovaciones, en los servicios se puede observar la inclusión de los usuarios o afectados en los equipos profesionales, la creación de contratos de cuidado

Cuadro 1: Diferencias entre los modelos de rehabilitación, integración y recuperación

\begin{tabular}{|c|c|c|c|}
\hline & Rehabilitación & Integración & Recuperación \\
\hline $\begin{array}{l}\text { Disciplinas } \\
\text { relacionadas }\end{array}$ & $\begin{array}{l}\text { Aprendizaje social, } \\
\text { psicología, psiquiatría social }\end{array}$ & $\begin{array}{l}\text { Derechos ciudadanos } \\
\text { procesos estigmatizadores } \\
\text { antropología, sociología }\end{array}$ & $\begin{array}{l}\text { Psicología narrativa y } \\
\text { cultural, antropología } \\
\text { social y cultural. }\end{array}$ \\
\hline Enfoque & Social e individual & Social y político & $\begin{array}{l}\text { Subjetivo, experiencias } \\
\text { personales, sociocultural. }\end{array}$ \\
\hline Objetivo & $\begin{array}{l}\text { Aprendizaje de conductas } \\
\text { reforzantes socialmente }\end{array}$ & $\begin{array}{l}\text { Ciudadanía, integración } \\
\text { social }\end{array}$ & $\begin{array}{l}\text { Construcción } \\
\text { identidad coherente, } \\
\text { logro del significado } \\
\text { personal. }\end{array}$ \\
\hline $\begin{array}{l}\text { Tipo de } \\
\text { intervención }\end{array}$ & $\begin{array}{l}\text { Programas de carácter } \\
\text { conductual }\end{array}$ & $\begin{array}{l}\text { Gestión sociosanitaria, } \\
\text { programas de } \\
\text { sensibilización e } \\
\text { información, programas } \\
\text { laborales y residenciales }\end{array}$ & $\begin{array}{l}\text { Influencia del entorno } \\
\text { relaciones informales o } \\
\text { asistemáticas, recursos } \\
\text { para construir } \\
\text { significados, denuncia. }\end{array}$ \\
\hline Perspectiva & Molecular & Molar & Molar \\
\hline $\begin{array}{l}\text { Evaluación e } \\
\text { Investigación }\end{array}$ & $\begin{array}{l}\text { Esencialmente } \\
\text { cuantitativa: psicométríca } \\
\text { y observacional }\end{array}$ & Mixta & $\begin{array}{l}\text { Esencialmente } \\
\text { cualitativa, experiencia } \\
\text { subjetiva, narrativa. }\end{array}$ \\
\hline Protagonistas & $\begin{array}{l}\text { Profesionales del área de } \\
\text { la psiquiatría y psicología }\end{array}$ & $\begin{array}{l}\text { Asociaciones de familiares, } \\
\text { grupos políticos }\end{array}$ & $\begin{array}{l}\text { Familiares, pacientes, } \\
\text { afectados en general, } \\
\text { clientes, supervivientes. }\end{array}$ \\
\hline
\end{tabular}


en los cuales los afectados negocian con los profesionales el tratamiento, nuevos equipos interdisciplinares y comunitarios como los assertive outreach, crisis resolution/home treatment team también comparten el espíritu de la aproximación a la recuperación en salud mental (UK, 1999). También podemos encontrar una creciente participación de los usuarios y afectados en las comisiones políticas que toman decisiones en la gestión del sistema de salud mental.

En América Latina, donde como hemos visto la reforma psiquiátrica y la normalización de los servicios de salud mental no está concluida, y en España, en la cual los procesos de reforma psiquiátrica han sido muy heterogéneos, no se han hecho visibles con claridad innovaciones como las señaladas anteriormente en el diseño y la gestión de los servicios. Sin lugar a dudas, el todavía incipiente movimiento de usuarios (que no de familiares el cual es más fuerte), la necesidad de un cambio de ideología en los profesionales en salud mental y desde luego, unos presupuestos en salud mental desiguales no permiten, hasta este momento, una transformación clara de los servicios de salud mental hacia los presupuestos de la recuperación. Es segura la llegada y la inclusión de estas ideas en nuestros sistemas de salud mental. Sin embargo, predecir el momento resulta imposible debido a las razones señaladas anteriormente.

Resumo en cuatro ideas las razones por las que considero esencial tomar en cuenta las investigaciones en la recuperación de las personas con TMG. Primero, estas investigaciones nos permiten descubrir qué recursos utilizan las personas con TMG para enfrentarse a la enfermedad mental desde su propia voz, más allá de los resultados que nos puedan ofrecer otros instrumentos analíticos clásicos como inventarios o técnicas observacionales. Segundo, la exploración de los caminos recorridos por las personas que sufren TMG para lograr dar sentido a sus vidas, al fin y al cabo, nos remite a la búsqueda de sentido a la vida de todos los seres humanos. Por lo tanto, estas investigaciones nos están hablando de las condiciones para la salud, entendida de forma global en todos los seres humanos. Tercero, estas investigaciones nos posibilitan estudiar cómo las personas se apropian y elaboran los significados y las herramientas culturales que los contextos socio-sanitarios ofrecen (Saavedra, 2009). Por ello, podríamos decir que el enfoque de la recuperación ofrece un nuevo campo en el cual analizar las relaciones entre mente y cultura. Cuarto, desde una perspectiva histórica, el concepto de recuperación puede ser comprendido como un nuevo marco ideológico a partir del cual se pueden construir y diseñar los servicios de salud mental. Especialmente en los países anglosajones, el concepto de recuperación es hoy en día un vector fundamental en el diseño y organización del trabajo de los profesionales de salud mental (The Future Vision Coalition, 2009) y constituye un horizonte cercano para los servicios de salud mental de América Latina, España y Portugal.

He dividido mi exposición de los resultados de las últimas investigaciones en el campo de recuperación en tres apartados y unas conclusiones. En el primer apartado, me ocuparé de la re-construcción de la identidad y del control de la propia vida de las personas con TMG en recuperación. Seguidamente, me centraré en las relaciones personales y el sentido de comunidad. En el tercer apartado, me adentro en el mundo de los recursos materiales, trabajo y otras actividades sociales. Por último, ofrezco una síntesis y unas reflexiones finales en el apartado de conclusiones. 


\section{Recuperación, identidad y control sobre la propia vida}

Numerosos autores (Mezinna et al., 2006; Roe, Davidson, 2005; Sells, Stayner, Davidson, 2004) han observado transformaciones importantes en las experiencias subjetivas de las personas con TMG tras un proceso de recuperación exitoso. Uno de los aspectos más importantes es la recuperación de la sensación de control sobre la propia vida y la capacidad de tomar decisiones. Es decir, la posibilidad de algún grado de autodeterminación por parte de las personas que sufren TMG. Un ámbito de estudio respecto a la sensación de control es su relación con el tratamiento psicofarmacológico. Jenkins y Carpenter-Song (2006), en un estudio sobre la experiencia subjetiva de noventa personas con TMG ambulatorios, diagnosticados con esquizofrenia o trastornos esquizoafectivos, encuentran, aproximadamente en la mitad de las narrativas de éstos, que no sólo el efecto de la medicación es importante, sino que el trabajo personal de enfrentarse a la enfermedad es esencial en el proceso de recuperación. Aquellos pacientes que perciben 'poder personal y control' declaran que aunque la medicación les ha ayudado, ellos han tenido que realizar un trabajo personal para lograr una mejora sustancial en su situación. Jekins y CarpenterSong (2006) consideran el énfasis en el progreso mediante el esfuerzo personal, es decir, la capacidad de agencia, como uno de los indicadores de superación en las narrativas analizadas.

Mezinna y colaboradores (2006) y Mancini (2007) afirman que muchas personas con TMG, entrevistadas en sus investigaciones, expresan que el control sobre el tiempo y el espacio en su vida cotidiana fueron los desencadenantes de la recuperación de su estabilidad emocional y funcionamiento. Así, la importancia de la búsqueda de espacios sociales que resulten seguros es esencial en los procesos de recuperación. En el estudio de Mancini (2007), los pacientes identifican distintas áreas de recuperación. Primero, desarrollar la competencia a través de experiencias de control. De este modo, los pacientes se comprometen en actividades significativas, tales como laborales, educativas, voluntarias o en el hogar, que desarrollen la percepción de la competencia y la agencia. Es necesaria la búsqueda por parte del paciente de actividades que se ajusten a su situación. La selección de una actividad inapropiada provocará un resultado contraproducente. Segundo, los participantes del estudio de Mancini identificaron el apoyo de los profesionales como vital en el proceso de recuperación. Los participantes definieron el apoyo profesional como afectuoso, respetuoso y constante en su apoyo. Los participantes declararon que los profesionales que los ayudaron creyeron en sus habilidades para recuperarse y les proveyeron de esperanza y fuerza. Mancini sitúa el apoyo profesional dentro de los recursos del incremento de la auto eficacia como un fenómeno de persuasión social. La tercera área en la que los participantes estuvieron de acuerdo en su importancia en la recuperación fue la autoayuda y el apoyo mutuo entre los pacientes. La autoayuda y el apoyo de otras personas con TMG proveen a muchos participantes de apoyo mutuo y modelos de recuperación. En este sentido, la creación de una comunidad de residentes en las casas hogares $\mathrm{u}$ otros recursos residenciales de apoyo son contextos privilegiados para intervenir.

El contexto de la vida cotidiana ha sido identificado por diversos investigadores como esencial para que los pacientes adquieran algún grado de control sobre sus vidas (Saavedra, 
2009). Las rutinas diarias y los hábitos relacionados con el hogar no sólo acercan a los pacientes a una vida normalizada, sino que ofrecen un espacio para que estos pacientes puedan tomar decisiones sencillas y básicas sobre la propia vida. Rutinas como levantarse, el aseo diario o las comidas pueden parecer insignificantes, pero estas actividades están formadas por pequeñas decisiones, como por ejemplo quedarse cinco minutos más en la cama, que proporcionan a las personas que padecen TMG las primeras experiencias de capacidad de agencia. Las rutinas diarias presentan unos guiones estables y seguros, es decir, dejan poco margen a la incertidumbre. Otra característica de estas rutinas es que en gran parte acontecen en un escenario interaccional de forma que requieren de la participación de otras personas. Estas características ayudan a que el paciente pueda tomar pequeñas decisiones mediante una negociación con los otros participantes que modifiquen los guiones, sin que estas decisiones impliquen un alto nivel de estrés. Este escenario se convierte en un contexto de aprendizaje muy importante.

A pesar del modelo narrativo cultural en el que el héroe logra un completo triunfo sobre la adversidad, por ejemplo una 'curación milagrosa' o un conmovedor triunfo frente a la enfermedad, en las narrativas de recuperación no se observan experiencias de curación o recuperación dramáticas. Encontramos, por una parte, historias marcadas por el abandono, la pérdida o el poder, en las cuales podemos hallar anhelos y expectativas para el futuro más o menos formuladas. Y por otra parte, hallamos historias de resistencia, de pequeños éxitos y alegrías, de búsqueda de sentido y de construcción de una identidad compatible con el mundo social mediante los contactos personales más cercanos y las actividades diarias. Son estas experiencias diarias, que pueden parecer insignificantes, las que sostienen el proceso de recuperación de los pacientes (Boydell, Gladstone, Volpe, 2003; Jenkins, Carpenter-Song, 2006; Ridgway, 2001; Roe, Davidson, 2005; Saavedra 2009).

Algunos autores (Boydell et al., 1999; Boydell, Gladstone, Volpe, 2003; Hermans, 1994) han sugerido que la falta de motivación generalizada de los pacientes con esquizofrenia, más que responder exclusivamente a un déficit cognitivo, es causa de una estrategia de defensa del estigma y de unas relaciones sociales fuertemente humillantes, de forma que por medio de un selectivo ocultamiento se protegen de un ambiente social hostil. Por lo tanto, la pasividad y el aislamiento, a menudo vistos como una pérdida de motivación, en muchos casos puede ser entendida como una activa, consciente y decidida acción. A partir de estas reflexiones se reconoce como esencial el facilitar a los pacientes espacios en los cuales retirarse y 'no hacer nada', si así lo deciden, al mismo tiempo que puedan evitar el aislamiento. En este sentido, en los recursos residenciales para enfermos que padecen TMG es muy importante la creación de espacios privados como dormitorios individuales o de lugares en los que se permita ausentarse 'espiritualmente' sin la obligación de realizar ninguna actividad.

Para los enfermos crónicos o de larga duración, la enfermedad o las experiencias en el entorno sanitario pueden ser los únicos materiales con los cuales construir una identidad adaptativa y entendible en la comunidad. En muchas ocasiones, la identidad del sujeto se ve engullida por el 'rol de enfermo' (McCay, Ryan, 2002). De este modo, el sujeto logra una identidad hasta cierto punto compartida en la comunidad, pero queda totalmente invalidada para presentarse como agente activo. En caso contrario, si la persona se niega a 
aceptar la etiqueta de enfermo mental, ésta puede verse marginalizada en mayor grado $y$, paradójicamente, ver confirmado el diagnóstico o la etiqueta de enfermo mental grave. Es indudable que una de las tareas esenciales a llevar a cabo por estas personas, las cuales no sólo han sido diagnosticadas con un TMG sino que presentan una experiencia de vida que se reduce casi exclusivamente a un contexto sanitario o de ayuda social, es negociar su identidad como enfermos mentales (Roe, Davidson, 2005). Jacobson (2001) enumera cuatro dimensiones en el proceso de recuperación: reconocer el problema, transformar la identidad, reconciliarse con el sistema y el contacto con otras personas. Jenkins y Carpenter-Song (2006) lo dicen de otra forma al indicar que un acercamiento a las experiencias de la enfermedad mental desde un 'modelo negociador', en contraste con un modelo que considere la etiqueta de enfermo mental como enemiga de la identidad, implica que la enfermedad y la identidad actual deben redefinirse en relación una a la otra.

El concepto que describe esta especie de 'darse cuenta' (insight) de los problemas psiquiátricos que se padecen es 'conciencia de enfermedad'. Algunos pacientes psiquiátricos, especialmente los esquizofrénicos, se han considerado como incapaces de dar cuenta de sus problemas y de los déficits psicológicos con coherencia. Sin embargo, la conciencia o inconsciencia de la enfermedad no es simplemente una cognición aislada, sino también un elemento de una historia vital y una comprensión narrativa de la vida del paciente (Lysaker et al., 2002). La conciencia o negación de la enfermedad está embebida en una gran historia que se encuentra inseparablemente unida a una gran variedad de sucesos pasados como de futuros sueños y expectativas. La aproximación a la conciencia o inconciencia de enfermedad desde el conocimiento o desconocimiento, acuerdo o desacuerdo de determinados síntomas o características de la enfermedad, impide la valoración de determinados hechos que pueden ayudar a comprender al paciente y a diseñar, de la mejor manera posible, los planes individuales de rehabilitación. Reformulando la definición clásica de conciencia de enfermedad, podríamos decir que una adecuada conciencia de enfermedad no es más que un uso adecuado del discurso profesional de forma que describiendo los problemas por medio de conceptos y argumentos compartidos socialmente no incapacite a la persona como sujeto activo, ni anule otras dimensiones de la identidad de éste. Esta tarea es muy compleja y requiere de importantes recursos cognitivos. Sells, Stayner y Davidson (2004) denominan como tácticas lingüísticas a aquellas estrategias discursivas utilizadas por los pacientes por las cuales transforman conceptos de carácter pasivo o peyorativo en atributos activos y positivos. Esta transformación del contenido semántico de los conceptos se puede lograr mediante la introducción de nuevos significados a conceptos preexistentes o mediante la reinterpretación de éstos. Estos autores nos ofrecen el ejemplo de un paciente que se autodenomina simple porque "yo hago las cosas simples. Yo no armo un lío por cosas no importantes. Yo soy pacífico". En este sentido, Mezinna y colaboradores (2006) afirman, a partir de numerosas investigaciones mediante entrevistas a pacientes, que las estrategias para negociar con el estigma que genera la enfermedad van desde el mostrarse como enfermo mental, enfatizando algunos componentes específicos del contenido semántico de este concepto como el de 'problemas para aprender' o 'malo de los nervios', hasta la lucha por los propios derechos como enfermo y persona. La lucha y la reivindicación de los propios derechos no sólo es un medio de incrementar las 
oportunidades de acceso a recursos sociales sino una forma de ir más allá de la simple identidad como enfermo mental. Jenkins y Carpenter-Song (2006) afirman que los pacientes que disfrutan de un proceso de recuperación exitoso desarrollan estrategias narrativas que oponen un nuevo yo recuperado al antiguo yo enfermo. Jekins y Carpenter-Song (2006) denominan este proceso como proceso de normalización, es decir, como un proceso que sitúa la enfermedad dentro de un concepto del yo integrado y que posibilita el contacto con el mundo exterior.

La palabra esquizofrenia, dentro de los TMG, despierta miedos y prejuicios en nuestra sociedad. Esta imagen tenebrosa de la esquizofrenia viene unida al pesimismo de la medicina oficial respecto al curso de la esquizofrenia. Todo ello a pesar de que numerosos resultados indican que una parte importante de los pacientes esquizofrénicos terminan disfrutando de una recuperación significativa (Jenkins, Carpenter-Song, 2006). Sin lugar a dudas, los pacientes deben rechazar esta imagen de la esquizofrenia que parece condenar de por vida a una parte importante de la población. Deben rechazarla, como decíamos antes, sin alejarse de una concepción socialmente aceptada de la enfermedad. Ahí radica la piedra angular de un proceso de recuperación exitoso. En este sentido, como cúspide de la pirámide de la recuperación es necesario crear larger life frames (Sells, Stayner, Davidson, 2004). La creación de estos marcos o narrativas supone re-elaborar la historia personal de los pacientes y dotarla de sensación de temporalidad, la construcción de campos de intersubjetividad y la elaboración de una posición activa dentro del mundo (Roe, Davidson, 2005; Sells, Stayner, Davidson, 2004). Reelaborar la historia de vida implica volver a seleccionar los eventos del pasado en una relación positiva con el presente haciendo posible la integración del pasado con los problemas actuales y una proyección hacia el futuro. Activar un plan de futuro requiere que los temas de pasividad de las narraciones personales se transformen en temas de agencia, así como integrar los distintos momentos de la vida (momentos de debilidad, de fuerza y motivación) en una historia lo suficientemente amplia que los integre. Los problemas traumáticos e inesperados de la enfermedad deben ser encauzados dentro de la historia vital por medio de términos favorables y conectados a un significado global del pasado y del presente. La construcción de campos de intersubjetividad puede ser entendida como nurturing psychological space (Sells, Stayner, Davidson, 2004) en la cual los pacientes pueden redefinir su identidad en una dirección positiva. Desde la perspectiva recuperadora se hace énfasis en la necesidad de una narración vital entendible socialmente y una identidad coherente y orientada hacia el futuro. En esta línea, otros autores han afirmado que los servicios sanitarios y sociales deben de estar diseñados para poder facilitar las experiencias y el apoyo necesario para que los pacientes puedan transformar sus narrativas (Holma, Aaltone, 1997; Roe, Davidson, 2005).

En un intento de síntesis Spaniol y colaboradores (2002) sugieren cuatro fases del proceso de recuperación. En la primera fase del proceso, alrededor de la primera crisis, la persona se encuentra sobrepasada física y psicológicamente por la experiencia. La persona intenta controlar y comprender lo que está sucediendo. Pero se siente confuso y desconectado de los demás. Padece una importante pérdida de la auto eficacia y teme un descenso generalizado del control de las experiencias internas y externas. Las personas son incapaces de articular claramente sus objetivos y éstos no están bien anclados en la realidad. En la 
segunda fase, las personas, más allá de la fase aguda de la enfermedad, desarrollan una explicación de lo que está sucediendo a menudo apoyadas por términos médicos o técnicos y afrontan la realidad de la naturaleza de larga duración o crónica de la enfermedad. Los pacientes pueden temer enfrentarse a nuevas actividades por miedo a perder la precaria estabilidad conseguida, pero ya disponen de estrategias para enfrentarse a la enfermedad y a los handicaps sociales relacionados. Esta etapa se caracteriza por la construcción del sentimiento de fuerza y confianza en la habilidad de actuar por los propios intereses. En la tercera fase, la persona ha construido un yo fuerte y estable que puede asumir los roles de la enfermedad. Se observa en esta etapa confianza y auto eficacia sobre el control de su vida. Las personas en esta etapa han aprendido a tener una vida satisfactoria a pesar de las dificultades y utilizan estrategias y habilidades eficaces en la lucha contra la enfermedad. La última etapa es descrita por estos autores como la construcción de una identidad en la que la enfermedad ocupa un espacio muy reducido sin que interfiera lo más mínimo en el disfrute de una vida productiva y satisfactoria.

\section{Recuperación, relaciones personales y comunidad}

El concepto de recuperación se extiende más allá de la reconstrucción y la negociación de la identidad a través de un trabajo personal. El concepto de recuperación engloba los procesos de negociación de la nueva identidad de los pacientes dentro de los contextos culturales y las relaciones sociales. Así, los contextos sociales se convierten en la llave que permite construir la identidad de las personas con TMG más allá de la enfermedad (Jenkins, Carpenter-Song, 2006; Mezinna et al., 2006). El proceso de recuperación engloba a muchas personas: familiares, amigos, profesionales, etc. Sin el concurso de estas personas no es posible ningún proceso de recuperación. Sólo a través de las relaciones personales y de los intercambios sociales en comunidad, el paciente puede adquirir una percepción de sí mismo normalizada. O lo que es lo mismo, sólo a través de la mirada de los otros, podemos construir una identidad compartible.

Mejorar las redes sociales de los pacientes significa restaurar las relaciones familiares y fomentar las redes alternativas. Un objetivo necesario, íntimamente unido a los contextos sociales en los que se desenvuelven los pacientes, es la sensación de pertenencia. Nos referimos a la sensación de estar compartiendo una serie de experiencias comunes, de encontrase en común unión, a la identificación con un grupo social o comunidad con los cuales se comparte una serie de valores, experiencias o características positivas. Es decir, una sensación de pertenencia voluntaria de la que nos sintamos orgullosos, la creación de un 'nosotros' que ayude a explicarnos un 'yo'. Quizás uno de los contextos donde la sensación de pertenencia se hace más evidente y necesaria sea el del propio hogar. En las investigaciones realizadas por Mezzina y colaboradores (2006) se encuentran algunas definiciones de hogar por parte de los pacientes. Mientras que alojamiento significaba para éstos 'un espacio privado que saca a la gente de la calle', la sensación de hogar fue descrita además como la oportunidad para la expresión personal y como un lugar seguro desde donde explorar el amplio mundo social: "La importancia de tener un hogar seguro, base o plataforma de la recuperación, se hizo muy visible y tangible en estas entrevistas. El hogar también fue 
descrito como un lugar para el crecimiento y el desarrollo, un lugar para la práctica y el crecimiento de la autodeterminación del yo, un vehículo para equilibrar lo privado y las demandas de la vida social" (p.70).

En relación con la influencia de los recursos residenciales, numerosas investigaciones señalan que éstas logran mayor estabilidad psicopatológica y salud física en los pacientes (Dorvil et al., 2005; Jones, Chesters, Flechter, 2003; Rössler et al., 1999). Dorvil y colaboradores (2005) indican que los recursos residenciales ofrecen espacios libres de juicios morales donde la experiencia de la enfermedad es aceptada. La presencia continua de profesionales en un contexto no clínico funciona como constante apoyo emocional. Los pacientes pueden comentar y discutir en todo momento con otros pacientes y profesionales las características de los síntomas y las experiencias comunes de enfermedad. Por lo tanto, los profesionales ayudan a los pacientes a identificar sus síntomas, proveen de habilidades para controlar éstos y fomentan recursos cognitivos y emocionales para reducir la ansiedad (Saavedra, 2009). Además, otros pacientes que se encuentran en un mejor estado psicopatológico pueden funcionar como ayuda en las situaciones de descompensación de sus compañeros. En cualquier caso, la recuperación no supone un ajuste pasivo a la experiencia de la enfermedad, sino que debe implicar un afrontamiento activo de las dificultades que derivan de los síntomas y del estigma asociado al diagnóstico. Los cuidadores formales pueden ayudar en los recursos residenciales a que los pacientes tomen cada vez mayor responsabilidad de su estado y se conviertan en promotores de su propia salud.

Unido a la sensación de pertenencia aparece otro importante sentimiento: la responsabilidad respecto a los otros, respecto a nuestros semejantes. Este es un aspecto al que se le ha prestado poca atención en relación con las personas que padecen TMG. Sin embargo, como muy bien nos recuerdan Jenkins y Carpenter-Song (2006), el apoyo social es un proceso recíproco. En las entrevistas que estos autores realizaron a pacientes de esquizofrenia sobresalieron con fuerza las actividades de cuidado que ofrecían los pacientes a otras personas. El ámbito de estas actividades de cuidado era muy amplio y variaba desde el simple apoyo ocasional a compañeros o amigos mediante la identificación de síntomas al cuidado durante años de los padres. Estas actividades son esenciales y merecen mayor atención y además deben ser fomentadas y reconocidas. Primero, porque suponen ir más allá de la identidad como enfermo, hasta el punto que se sitúa en el lado opuesto, es decir como agente de salud; además, se potencia la identidad de las personas como agentes activos reconocidos. Segundo, porque se posiciona a la persona no como paciente, sino como experto en sus experiencias de enfermedad, el cual puede apoyar y acompañar a otras personas en sus momentos críticos. Estas actividades de cuidado por parte de los pacientes también se pueden extender hacia los profesionales de salud. La pertenencia a un grupo y la responsabilidad hacia los semejantes otorga a los pacientes una muy positiva sensación de dignidad, seguridad y estabilidad, ya que implica no sentirse solo en la lucha contra el estigma y la enfermedad, y abre la puerta a la comprensión del concepto de ciudadanía. La conquista del estatus de ciudadano para el enfermo mental ha sido uno de los logros de la salud mental comunitaria. Sentirse miembro de un grupo social y responsable de la calidad de vida de otros, familia, compañeros, etc., es condición necesaria para reconocer la existencia de instancias superiores que al mismo tiempo sean responsables de 
nuestra situación social y a las cuales podemos reclamar nuestros derechos, ya que éstos sólo emergen de nuestra pertenencia a una comunidad. De esta forma, los esfuerzos individuales para conseguir una mejor calidad de vida se transforman en intereses colectivos y suponen una reformulación del estatus de los pacientes para convertirlos en ciudadanos.

En la esfera interpersonal, Mezzina y colaboradores (2006) describen tres grandes cauces por los cuales las relaciones con otras personas pueden facilitar la recuperación. Las personas con TMG afirman que una manera mediante la cual se les puede ayudar es 'estando allí'. Éstas se refieren con este término, por una parte, a los profesionales de salud mental que trabajan directamente con ellas y pueden acompañarlas sin exigir ni preguntar nada, permitiendo una positive withdrawal. Nos referimos a un espacio de libertad para una especie de retirada espiritual o de inacción. Sin lugar a dudas, el primer paso para la mejora del funcionamiento social de los pacientes es un sentimiento de seguridad y libre de exigencia en un espacio social. El estar sentado con un paciente en el mismo sillón sin hablar durante una hora es un ejemplo de ello. Muchas personas relatan experiencias de este tipo como muy significativas. En relación con la familia, 'estando allí' significa la continuidad y persistencia del apoyo de los allegados en la historia personal de los pacientes, a pesar de las difíciles fases de la enfermedad. También nos informan que además se puede 'hacer más'. Los familiares pueden hacer más realizando esfuerzos extras para exigir servicios más efectivos y para reivindicar derechos por sus allegados, pueden hacer más prestando algo de dinero, disculpando errores o sacrificándose en algunos aspectos por sus allegados. Los profesionales de salud mental pueden hacer más yendo más allá del simple rol profesional en la creación de contactos personales y en circunstancias extraordinarias como en crisis psicóticas apoyando a los pacientes a pesar de las posibles reacciones negativas de éstos. Un tercer tipo de relaciones implica 'hacer algo diferente'. Hacer algo diferente implica relacionarse con los usuarios desde dimensiones de la identidad que vayan más allá de los atributos de 'enfermo mental'. Hacer algo diferente es relacionarse con los pacientes desde las habilidades, capacidades de las que disfrutan. Hacer algo diferente significa no ajustarse a las definiciones de la enfermedad de las instituciones, no limitarse a los roles y reglas que determinan éstas en las relaciones personales.

\section{Recuperación, recursos materiales, trabajo y otras actividades sociales}

Es indudable que la primera condición para la recuperación es el disfrute de las condiciones mínimas de vida: un alojamiento digno, alimentación, higiene y asistencia sanitaria. El primer objetivo de los recursos residenciales para personas que padecen TMG es satisfacer las necesidades básicas de éstos.

Sin embargo, como es lógico, no todo acaba ahí. Un marco fundamental en la recuperación es la dimensión laboral. Se encuentra ampliamente demostrado que los roles laborales son fundamentales en las sociedades occidentales. En la cultura occidental, el trabajo es altamente evaluado y es considerado una fuerza socialmente integradora. Sin embargo, la gran mayoría de las personas con enfermedad mental son excluidas del mundo laboral. El empleo puede servir como un elemento normalizador para individuos estigmatizados por su enfermedad mental que están desempleados y han perdido los roles 
correspondientes. A través del trabajo, los individuos pueden obtener estructura diaria y pueden desarrollar una red social de contactos interpersonales. Lysaker y colaboradores (1995) y Lysaker y France (1999) remarcan la importancia del rol laboral en la construcción de una identidad coherente y socialmente aceptada. Apuntan que los problemas cognitivos no son los únicos que tienen que afrontar estos pacientes para lograr una vida activa laboralmente. Una construcción de la identidad defectuosa impide que se puedan activar planes de acción significativos estables, imprescindible para el mundo laboral. Por lo tanto, es imprescindible posibilitar muy tempranamente la integración de los roles laborales en la débil identidad original del sujeto. Bebout y Harris (1995) aportan dos consideraciones muy interesantes. Por una parte, el refuerzo de la consolidación de la identidad como enfermo, es decir, aquello que llamamos como conciencia de enfermedad, puede provocar una hipertrofia de este posicionamiento, lo cual va a dificultar la construcción de otras posibles historias en las cuales el sujeto se describa bajo otros términos. Por otra parte, Bebout y Harris (1995) nos indican que la visión de la vulnerabilidad al estrés, como un rasgo esencial en la esquizofrenia, provoca que se actúe por parte de los profesionales con actitudes muy paternalistas, las cuales impiden el desarrollo integral del rol laboral en los enfermos. En cualquier caso, como afirman Mezzina y colaboradores (2006), ninguna actividad estructurada como un empleo puede mejorar la capacidad de agencia y lograr la recuperación si no es elegida y es significativa para la persona.

Es reseñable que el hecho de que el trabajo formal implique el pago de los impuestos facilita la integración de las personas como ciudadanos de pleno derecho y hace más fácil que las personas se consideren como tales. Uno de los aspectos positivos de las ocupaciones laborales es que proporcionan ingresos económicos. No podemos olvidar la dimensión como consumidor de las personas en el proceso de recuperación. Generalmente esta dimensión es olvidada. El consumo es una de las actividades normalizadoras para las personas que padecen TMG. Una persona sin una mínima capacidad de consumo no puede integrarse socialmente. El consumo es una de las dimensiones más importantes y definidoras de nuestra sociedad (Saavedra, 2007). Tanto es así que no basta con tener una ocupación laboral, sino que es necesario poder acceder a los centros de consumo y disfrutar del derecho a la propiedad. Muchas de las personas con TMG disfrutan desde hace años de una actividad laboral estructurada, generalmente en alguna institución de apoyo, sin embargo permanecen completamente fuera de los contextos sociales de consumo. Y, hoy en día, mantenerse fuera de los contextos de consumo es sinónimo de marginación. Poder acercarse una vez al mes al centro de la ciudad para hacer algunas compras, visitar algún centro comercial o regalarse algún objeto son actividades de carácter integrador. Sin lugar a dudas, esta dimensión del proceso de recuperación requiere de mayor investigación.

La actividad laboral es esencial, pero no es la única que facilita la recuperación. La vuelta al contexto educativo tras un periodo de agravamiento de la enfermedad es una meta muy positiva en el proceso de recuperación. Otras actividades pueden ser la participación activa en asociaciones de enfermos y familiares en la lucha por la defensa de los derechos de los enfermos, en clubes deportivos, asociaciones de vecinos, sociales, etc. (Bebout, Harris, 1995). 


\section{Conclusiones}

Sintetizo lo dicho hasta ahora sobre el concepto de recuperación a partir de ocho premisas. Primero, la recuperación significa rescatar la esperanza. Empezar a recuperarse implica abandonar una concepción de la esquizofrenia o cualquier TMG como insuperable. Por lo tanto, la recuperación puede ser entendida como una ruptura o negación de un modelo de enfermedad. Segundo, implica un esfuerzo por lograr la comprensión y la aceptación. Tercero, la recuperación significa ir más allá de la identidad como enfermo y reivindica el reconocimiento de otras dimensiones de la identidad. Cuarto, supone un movimiento desde el aislamiento hacia el compromiso y a la participación activa en la vida. Quinto, es más un estilo de afrontamiento activo que un ajuste pasivo a las condiciones de la enfermedad. Sexto y muy importante, la recuperación es un viaje largo y complejo con idas y vueltas. Séptimo, implica abandonar la alienación y dirigirse hacia la conquista del sentido y el propósito en la propia vida. Y por último, la recuperación necesita del apoyo y de relaciones sociales positivas.

Tras la descripción de las dimensiones de la recuperación (ver Diagrama 1), no es difícil comprender la complejidad de la tarea de los profesionales e instituciones que trabajan en pos de la recuperación de las personas que padecen un TMG. Pero, sin lugar a dudas, la tarea más ingente, hasta el punto de poderse considerar como heroica, es la que debe realizar la persona que sufre un TMG. Esta persona debe luchar contra la misma enfermedad, contra los procesos de marginación asociados a la enfermedad y contra el estigma. Todo ello mientras se guarda el equilibrio de una identidad que pueda ser compartida en sociedad. En este sentido, los esfuerzos personales por la recuperación han sido denominados como esfuerzos morales (Kleinman, 1999), ya que implica la búsqueda de las condiciones intersubjetivas que puedan aliviar la opresión de la enfermedad y el estigma. En definitiva, la recuperación consiste en encontrar un lugar en el mundo.

A pesar de los intentos de dotar de coherencia interna a los procesos de recuperación, éstos evidencian en nuestra cultura occidental un conjunto de paradojas (Jenkins, CarpenterSong, 2006). Debemos tenerlas en cuenta ya que las personas que padecen TMG son conscientes de ellas. La paradoja más importante y que, de alguna manera resume todas demás, es la idea de la recuperación sin cura. A pesar de la visión esperanzadora que hemos expresado aquí y de los datos objetivos que contradicen la visión catastrofista de los TMG, muy pocos se atreverían a negar el carácter crónico de enfermedades como la esquizofrenia. Por lo tanto, muchas personas a pesar de la remisión de los síntomas y de una recuperación exitosa no podrán liberarse completamente de algún tipo de relación con la 'enfermedad'. A pesar de la mejoría, la toma de la medicación, en muchos casos de por vida, y el padecimiento de algunos efectos secundarios, por ejemplo de carácter sexual, obligará a enfrentarse a muchas personas con etiquetas como 'enfermo' o 'adicto' durante toda su vida.

La causa explicativa del 'desequilibrio bioquímico' de los TMG, la cual puede ser aceptada socialmente, libera de sentimientos de culpabilidad (incluidas familias) y protege a la identidad de las personas de ser invadidas por el rol de enfermo. Sin embargo, como es fácil de comprobar, no libera del estigma. Y no sólo eso, sino que puede conllevar a que las personas que padecen TMG se consideren como incapaces de intervenir en su propia vida 
Diagrama 1: Dimensiones y proceso de recuperación

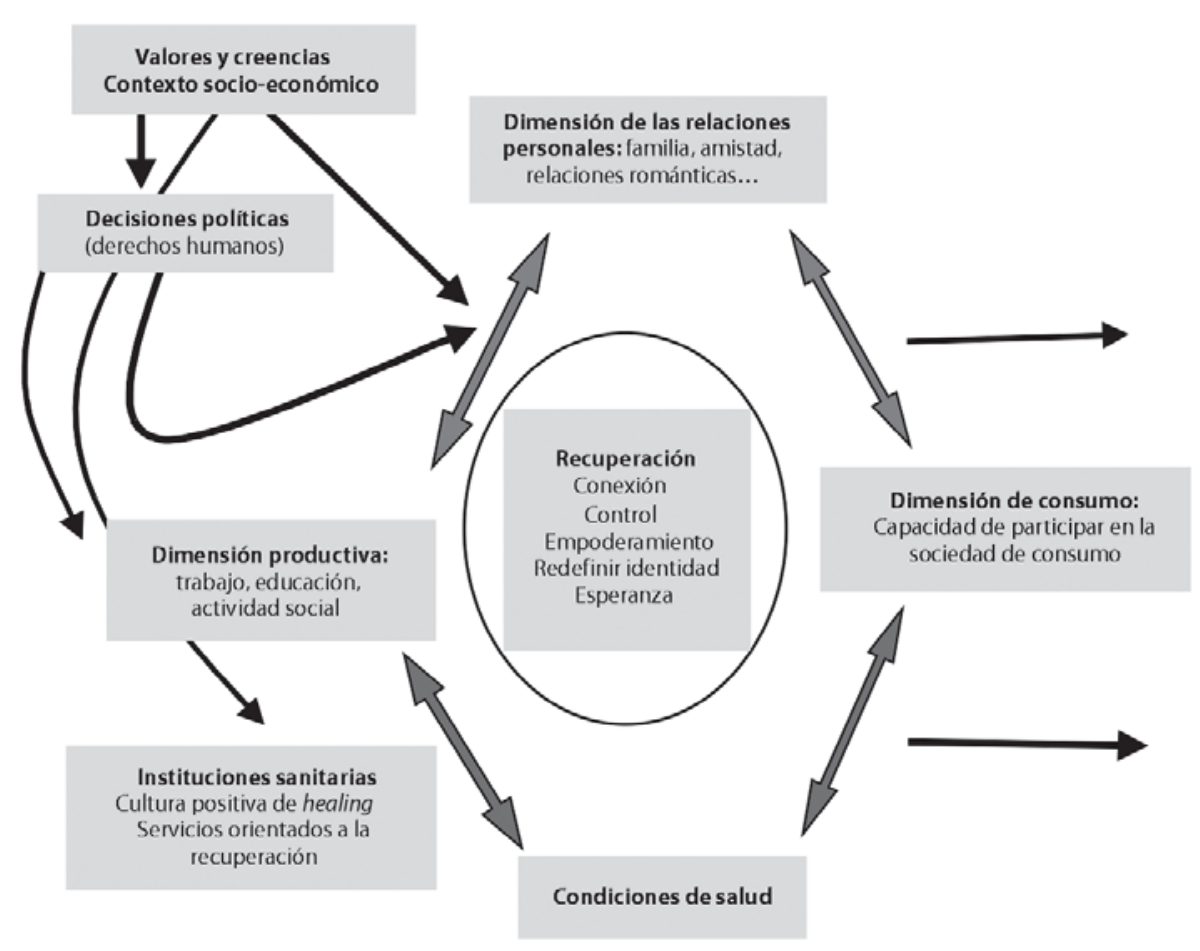

e influir en el curso de sus problemas al hacer depender su situación de una realidad que escapa a su campo de acción. Además, algunas investigaciones señalan que los profesionales que no entienden la experiencia de enfermedad, de acuerdo con una perspectiva psicobiológica, muestran mejor actitud hacia las personas que padecen trastornos mentales graves (Read, Haslam, 2005). De hecho, las personas con TMG tienden a dar cuenta de sus problemas de salud mental preferentemente acudiendo a factores autobiográficos y psicosociales (Saavedra, 2009). Sin lugar a dudas, este efecto ambivalente de las explicaciones biológicas de la enfermedad mental constituye una importante paradoja.

Es necesario hacer énfasis en la necesidad de intervenir en los contextos locales donde se desarrollan los procesos de recuperación. Es imprescindible trabajar, al mismo tiempo que con las personas que padecen TMG, con los prejuicios, las actitudes e injusticias económicas y políticas que contaminan las comunidades sociales. Los valores y creencias sociales y el sistema socio-económico dominante son realidades a tener en cuenta en los procesos de recuperación. Los procesos de recuperación son muy dependientes de los contextos sociales. Además, facilitar la recuperación de personas que padecen TMG requiere no focalizar el trabajo en exclusiva en estas personas, sino preocuparse por el diseño de las instituciones que las atienden. Es decir, se necesita investigar sobre la gestión de la salud mental y sobre la cultura profesional privilegiada en nuestras instituciones. Sin lugar a dudas, los esfuerzos que los sistemas de salud de América Latina están llevando a cabo por reformar la atención psiquiátrica van en esta dirección (Larrobla, 2007; Rodríguez, 2007; Nacimiento Alves, 2007; Tosta, 2009). 
Existe una acepción del concepto de recuperación que va claramente más allá de una simple visión rehabilitadora. El concepto dirige nuestra atención a la necesidad de que los 'pacientes' abandonen la posición de 'pacientes' y sean protagonistas de sus historias de recuperación en todos los niveles. Para ello, es imprescindible que sean parte de la toma de decisiones socio-políticas que les afectan en distintos niveles. La reforma psiquiátrica, basada en distintos modelos de rehabilitación psicosocial, se inició apoyándose fundamentalmente en la iniciativa y la fuerza de grupos de profesionales comprometidos. Por ejemplo, en Brasil por medio del Movimiento de los Trabalhadores en Saúde Mental y mediante experiencias e iniciativas espacialmente relevantes, como la intervención de la Casa de Saúde Anchieta, en Santos, en 1989 (Nacimiento Alves, 2007). En el futuro, las personas con TMG, de acuerdo con el concepto de recuperación y por medio de asociaciones de afectados, deben paulatinamente formar parte de los órganos de consulta y decisión relacionados con la gestión de la salud mental. Por ejemplo, en Inglaterra, numerosas asociaciones de personas con TMG participan activamente en comisiones oficiales. Sin lugar a dudas, este nuevo paso será generador de tensiones ya que reta el equilibrio profesional e institucional existente y será necesario diseñar espacios de colaboración entre profesionales, políticos y afectados. En mi opinión, considerar a una persona con TMG como un ciudadano de pleno derecho implica reconocerle capacidad de agencia e influencia en las decisiones que les afecta. De este modo, sólo pueden considerarse integradas las personas que operan dentro del sistema que produce valores, riqueza y reconocimiento social. Excluidos son aquellos que no participan de los intercambios sociales regulares.

Una verdadera reforma sanitaria, como la de la gestión de la salud mental, es imposible sin un cambio de premisas ideológicas y estructurales en las instituciones sanitarias. Por ello, cualquier cambio que no lleve consigo esa transformación no es más que responder a un reajuste del sistema a nuevas condiciones económico-sociales. Como muy acertadamente nos advierten Dorvil y colaboradores (2005, p.513) "la desinstitucionalización requiere una redefinición de la noción de integración, control y normalización, las cuales son centrales para las ciencias sociales".

\section{NOTAS}

${ }^{1}$ En esta y demás citas de otros idiomas, la traducción es libre.

2 Por ejemplo, The Hearing Voices Network (http://www.hearing-voices.org/information.htm). The Mindfreedom International Association (http://www.mindfreedom.org/join-donate) y La asociación Peoplewho (http://www.peoplewho.org).

\section{REFERENCIAS}

BEBOUT, Richard; Harris, Maxine. Personal myths about work and mental illness: response to Lysaker and Bell. Psychiatry, London, v.58, n.4, p.410-423. 1995.

BOYDELL, Katherine et al.

Making do on the outside: everyday life in the neighbourhoods of consumers. Psychiatric Rehabilitation Journal, Boston, v.23, n.1, p.11-17. 1999.
BOYDELL, Katherine; GLADSTONE, Brenda; VOLPE, Tiziana.

Interpreting narratives of motivation and schizophrenia: a biopsychosocial understanding. Psychiatric Rehabilitation Journal, Boston, v.26, n.4, p.422-426. 2003.

BROWN, George; RUTTER, Michael. The measure of family activities and 
relationship: a methodological study. Human Relation, London, n.19, p.241-63. 1966.

CALDAS DE ALMEIDA, José Miguel. Logros y obstáculos en el desarrollo de políticas y servicios de salud mental en los países de América latina y el Caribe después de la Declaración de Caracas. Conferencia dictada en la Conferencia Regional sobre la Reforma de los servicios de Salud Mental "15 años después de la Declaración de Caracas", OPS/OMS-MS/ Brazil, 7-9 nov. 2005. Brasília. 2005.

CIOMPI, Luc.

Toward a coherent multidimensional understanding and therapy of schizophrenia: converging new concepts. In: Strauss, J.J.; Boker, W.; Brenner, H.D. (Ed.). Psychological treatment of schizophrenia. Toronto: Hans Huber. p.48-62. 1987.

CSIP, RCPSYCH, SCIE.

Care Services Improvement Partnership; Royal College of Psychiatrists; Social Care Institute for Excellence. A common purpose: recovery in future mental health services. London: Care Services Improvement Partnership. (Joint Position Paper, 08). 2007.

DORVIL, Henri et al.

Housing as a social integration factor for people classified as mentally ill. Housing Studies, London, v.20, n.3, p.497-519. 2005.

HERMANS, Hubert.

Former crazies in the community. In: Dietz, M.; Prus, R.; Shaffir, W. (Ed.). Doing everyday life: ethnography as human lived experience. Toronto: Copp Clark Longman. p.354-372. 1994.

HOLMA, Juha; AALTONEN, Jukka.

The sense of agency and the search for narrative in acute psychosis. Contemporary Family Therapy, St. Louis, n.19, p.463-477. 1997.

JACOBSON, Nora.

Experiencing recovery: a dimensional analysis of recovery narratives. Psychiatric Rehabilitation Journal, Boston, v.24, n.3, p.248-256. 2001.

JACOBSON, Nora; CURTIS, Laurie. Recovery as policy in mental health services: strategies emerging from the States. Psychiatric Rehabilitation Journal, Boston, v .23, n.4, p.333-341. 2000.

JACOBSON. Nora; GREENLEY, Dianne. A conceptual model of recovery. Psychiatric Services, Arlington, v.52, n.5, p.688-688. 2001.

JENKINS, Janis; CARPENTER-SONG, Elizabeth. The new paradigm of recovery from schizophrenia: cultural conundrums of improvement without cure. Culture, Medicine and Psychiatry, Cleveland, v.29, p.379-413. 2006.
JONES, Rebeca; CHESTER, Janice; FLECHTER, Meredith.

Make yourself at home: people living with psychiatric disability in public housing. International Journal of Psychosocial Rehabilitation, London, v.7, p.67-79. 2003.

JUNTA DE ANDALUCÍA. Consejería de Salud. II Plan Integral de Salud Mental de Andalucía, 2008-2012. Sevilla: Consejería de Salud. Disponible en: http:// www.capitalemocional.com/Textos_Psico/ II\%20plan\%20de\%20salud\%20mental\%20and \%202008-2012.pdf. 2008.

JUNTA DE ANDALUCÍA.

Consejería de Salud. Sistema Sanitario Público de Andalucía. Plan Integral de Salud Mental de Andalucía, 2003-2007. Sevilla: Servicio Andaluz de Salud, Consejería de Salud, Junta de Andalucía. Disponible em: http://www.junta deandalucia.es/servicioandaluzdesalud/library/ plantillas/externa.asp?pag=../../publicaciones/ datos/172/pdf/Salud mental 2003-2007.pdf. 2003.

KLEINMAN, Arthur.

Moral experience and ethical reflection: can medical anthropology reconcile them?

Daedalus, New York, v.128, p.69-99. 1999.

\section{LARROBLA, Cristina.}

Unidades psiquiátricas en hospitales generales en América del Sur: contexto y panorama general de los últimos años. Revista de Psiquiatría del Uruguay, Montevideo, v.71, n.2, p.125-134. 2007.

\section{LIBERMAN, Robert.}

Coping and competence as protective factors in the vulnerability-stress model of schizophrenia. In: Goldstein, M.; Hand, I.; Hahlweg, K. (Ed.). Treatment of schizophrenia, Berlín: Springer. p.201-216. 1986.

LÓPEZ, Marcelino; LAVIANA, Margarita. Rehabilitación, apoyo social y atención comunitaria a personas con trastorno mental grave: propuestas desde Andalucía. Revista de la Asociación Española de Neuropsiquiatría, Madrid, v.27, n.99, p.187-223. 2007.

LYSAKER, Paul et al.

Insight and personal narrative of illness in schizophrenia. Psychiatry, Boston, v.65, n.3, p.197-206. 2002.

LYSAKER, Paul et al.

Social skills at work: deficits and predictors of improvement in schizophrenia. Journal Nervous Mental Disorder, Philadelphia, v.183, n.11, p.688-692. 1995.

LYSAKER, Paul; FRANCE, Catherine.

Psychotherapy as an element in supported 
employment for persons with severe and persistent mental illness. Psychiatry, Boston, v.62, n.3, p.209-221. 1999.

MANCINI, Michael.

The role of self-efficacy in recovery from serious psychiatric disabilities. Qualitative Social Work, Boston, v.6, p.49-74. 2007.

MCCAY, Elizabeth; RYAN, Kathy.

Conocer las necesidades emocionales del paciente. In : Zipursky, R.B.; Schulz, S.CH. (Comp.). Estados iniciales de la esquizofrenia. Barcelona: Ars Medica. p.107-128. 2002.

MEZZINA, Roberto et al.

Social nature of recovery: discussion and implications for practice. American Journal of Psychiatric Rehabilitation, Philadelphia, v.9, p.63-80. 2006.

\section{MIND.}

Life and times of a supermodel: the recovery paradigm for mental health. London: MindThink. (MindThink report 3). 2008.

NACIMIENTO ALVES, Domingo Sávio. La declaración de Caracas y la reforma psiquiátrica en Brasil. In: Rodríguez, J. (Ed.). La reforma de los servicios de salud mental: 15 años después de la Declaración de Caracas. Washington: OPS. 2007.

NUECHTERLEIN, Keith; DAWSON, Michael. A heuristic vulnerability/stress model of schizophrenic episodes. Schizophrenia Bulletin, Oxford, v.10, n.2, p.300-312. 1984.

READ, John; HASLAM, Nick.

Public opinions: bad things happen and can drive you crazy. In: Read, J; Mosher, L.R.; Bental, R.P. Models of madness. New York: Routledge. p.133-146. 2005.

RIGDWAY, Priscila.

Restoring psychiatric disability: learning from first person recovery narratives. Psychiatric Rehabilitation Journal, Boston, v.24, n.4, p.335-343. 2001.

RODRÍGUEZ, Jorge.

La atención a la salud mental en América Latina y el Caribe. Revista de Psiquiatría del Uruguay, Montevideo, v.71, n.2, p.117-124. 2007.

ROE, David; DAVIDSON, Larry,

Self and narrative in schizophrenia: time to author a new story. Medical Humanities, London, n.31, p.89-94. 2005.

RÖSSLER, Wulf et al.

Does the place of treatment influence the quality of life of schizophrenics?. Acta Psychiatrica Scandinavica, Aalborg, v.100, n.2, p.142-148. 1999.
SAAVEDRA, Javier.

Schizophrenia, narrative and change:

Andalusian care homes as novel sociocultural context. Culture, Medicine and Psychiatry, Cleveland, v.33, n.2, p.163-184. 2009.

SAAVEDRA, Javier.

La identidad social en la sociedad de consumo. Nómadas. Revista de Ciencias Sociales y Jurídicas, Madrid, v.16, p.363-380. 2007.

SAINSBURY CENTRE FOR MENTAL HEALTH. Implementing recovery: a new framework for organisational change. London: Sainsbury Centre for Mental Health. Disponible en: http:/ /www.centreformentalhealth.org.uk/pdfs/ implementing_recovery_paper.pdf. 2009.

SELLS, Dave; STAYNER, David; DAVIDSON, Larry.

Recovering the self in schizophrenia: an integrative review of qualitative studies. Psychiatric Quarterly, New York, v.75, n.1, p.87-97. 2004.

SHEPHERD, Geogg; BOARDMAN, Jed; SLADE, Mike.

Making recovery a reality. London: Sainsbury Centre for Mental Health. 2008.

SPANIOL, Leroy et al.

The process of recovery from schizophrenia. International Review of Psychitary, London, v.14, n.4, p.327-336. 2002.

THE FUTURE VISION COALITION.

A future vision of mental health. [S.l.]: The Future Vision Coalition. Disponible en: http://www.newvisionformentalhealth.org.uk/ A_future_vision_for_mental_health.pdf. 2009.

TOSTA, Manoel.

La reforma psiquiátrica brasileña: perspectivas y problemas. Salud Mental, Mexico, v.32, n.4, p.265-267. 2009.

UK.

United Kingdom. Department of Health. A national service framework for mental health: modern standards and service models. London: Department of Health. 1999.

VIZEU, Fabio.

The modern psychiatric institution from an organizational perspective. História, Ciências, Saúde - Manguinhos, Rio de Janeiro, v.12, n.1, p.33-49. 2005.

ZUBIN, Joseph; SPRING, Bonnie.

Vulnerability: a new view of schizophrenia. Journal of Abnormal Psychology, Washington, v.86, n.2, p.103-126. 1977. 